\title{
Textrode-enabled transthoracic electrical bioimpedance measurements - towards wearable applications of impedance cardiography
}

\author{
J. C. Marquez ${ }^{1,2,4}$, M. Rempfler ${ }^{2}$, F. Seoane ${ }^{1,2}$ and K. Lindecrantz ${ }^{1,3}$ \\ 1. School of Technology and Health, KTH Royal Institute of Technology, Huddinge, Sweden \\ 2. School of Engineering, University of Borås, Borås, Sweden \\ 3. Department of Clinical Science, Intervention, and Technology, Karolinska Institutet, Stockholm, Sweden \\ 4. E-mail any correspondence to: jcmr@kth.se
}

\begin{abstract}
During the last decades the use of electrical bioimpedance (EBI) in the medical field has been the subject of extensive research, as an affordable, harmless and non-invasive technology. In some specific applications, such as body composition assessment where EBI has proven a good degree of effectiveness and reliability, the use of textile electrodes and measurement garments have shown good performance and reproducible results. Impedance cardiography (ICG) is a modality of EBI that can benefit from the implementation and use of wearable sensors. ICG is based on continuous impedance measurements of a longitudinal segment across the thorax taken at a single frequency. The need for a specific electrode placement on the thorax and neck can be easily ensured with the use of a garment with embedded textile electrodes, also known as "textrodes." The first step towards the implementation of garment-based ICG is to determine the quality of ICG measurements with textile sensors to allow estimation of fundamental ICG parameters. In this work, the measurement performance of a 2-belt set with incorporated textrodes for thorax and neck is compared against ICG measurements obtained with $\mathrm{Ag} / \mathrm{AgCl}$ electrodes. The analysis is based on the quality of the fundamental ICG signals $(\Delta \mathrm{Z}, \mathrm{dZ} / \mathrm{dt}$ and $\mathrm{ECG})$, systolic time intervals and other ICG parameters. The results indicate the feasibility of using textrodes for ICG measurements with consistent measurements and relatively low data dispersion. Thus, enabling the development of measuring garments for ICG measurements.
\end{abstract}

Keywords: Bioimpedance, impedance cardiography, ICG, textile electrodes, textrodes

\section{Introduction}

The implementation and use of textile technology and garments for electrical bioimpedance (EBI) measurements have been studied before, with reported results that are consistent and encouraging. Nonetheless, applied research regarding measurement performance of the garments is still necessary.

Previous studies of bio potential sensing $[1,2]$ and EBI for body composition analysis have shown the feasibility of using, textile electrodes, typically called "textrodes" [3, 4]. In impedance cardiography (ICG) the use of a measurements garment with integrated textrodes would facilitate continuous monitoring of cardiac activity.
The usefulness of ICG has been questioned by many but it is endorsed by the U.S. Department of Health \& Human Services through the Centers for Medicare \& Medicaid Services (CMS) [5] for a number of clinical uses, patient management, and monitoring applications. In addition the particular and distinctive capability of ICG to provide beatto-beat cardio-vascular information non-invasively opens several monitoring scenarios.

Many of the applications endorsed by the CMS would benefit greatly from a wearable-ICG measurement system. For example, the optimization of fluid management in patients with congestive heart failure based on patient monitoring at home.

In a preliminary study [6], ICG and ECG signals were recorded with the textile belts showing waveforms similarities. For this study the analysis of the textile belts was extended to a performance comparison of some parameters calculated from the characteristic points of the recorded signals i.e. heart rates, peak first time derivative $(\mathrm{dZ} / \mathrm{dt})$, time intervals, and stroke volume $(\mathrm{SV})$. The comparison was done using thoracic electrical impedance (TEB) measurements obtained with $\mathrm{Ag} / \mathrm{AgCl}$ electrodes as reference.

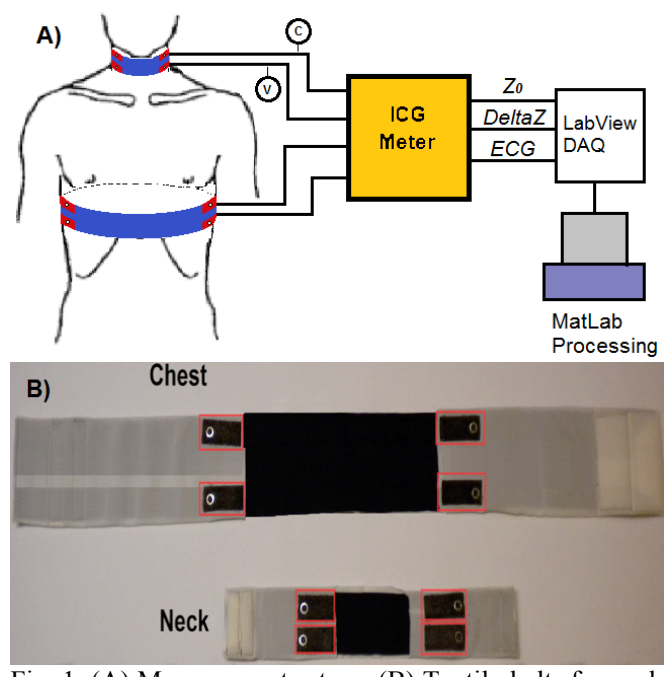

Fig. 1 (A) Measurement set-up. (B) Textile-belts for neck and chest with the four textrodes highlighted in red. 


\section{A. Impedance Cardiography}

The biophysical properties of blood, such as a conductivity twice that muscle tissue and several times higher than most other types of tissue [7, 8], produces cardio-related impedance changes that are easily recorded. Important parameters reflecting cardiac activity can be obtained from thoracic electrical bioimpedance measurements such as impedance change, $\Delta \mathrm{Z}$, and a first time derivative, $\mathrm{dZ} / \mathrm{dt}$. Using the correlation found by Lababidi et al [9] between the ICG waveforms and mechanical events of the cardiac muscle, characteristic points from the ECG and ICG signals can be used to estimate systolic time intervals and other clinically important hemodynamic indices.

ICG technology however, has not reached full acceptance among practitioners for the diagnosis and monitoring of hemodynamic activity in patients with cardiovascular conditions. Under clinical criteria, this technology has not been acknowledged as an established method, primarily because it has presented an unpredictable accuracy in the hemodynamic estimation for patients with severe heart disease or are critically ill [10] and secondly because of controversy about the physiological and anatomic origin of $\mathrm{dZ} / \mathrm{dt}$ [11].

Nonetheless the United States Department of Health and Human Services (USHHS) endorses the use of TEB for specific cases of the following medical conditions [5]:

- Differentiation of cardiogenic from pulmonary causes of acute dyspnea.

- Optimization of atrioventricular (A/V) interval for patients with $\mathrm{A} / \mathrm{V}$ sequential cardiac pacemakers.

- Monitoring of continuous inotropic therapy for patients with terminal congestive heart failure.

- Evaluation for rejection in patients with heart transplant as a predetermined alternative to myocardial biopsy.

- Optimization of fluid management in patients with congestive heart failure.

\section{Materials and methods}

\section{A. Textrode Belt}

A set of two custom-made textile belts for neck and chest have been manufactured with neoprene and synthetic wrap knitted fabric at Swedish School of Textiles at the University of Borås. Four textrodes were embedded in the belts following a lateral spot electrode array [12] as is shown in Fig. 1.B. The textrodes had a contact area each of approximately $13.5 \mathrm{~cm}^{2}$ and are made with conductive Velcro with loops made of silver coated fibers. Snapbuttons were used to enable the connection with the ICG measurement instrumentation. The adjustment of the belts and the correct positioning of the textrodes were achieved through Velcro fasteners and the stretchy properties of the belts materials.

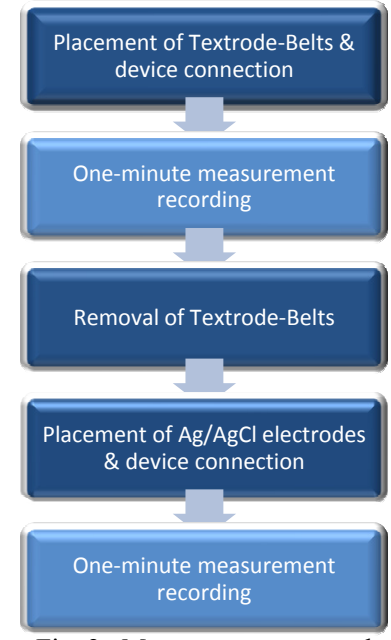

Fig. 2 Measurement protocol

\section{B. Ag/AgCl Electrolytic Gel Electrode}

Red Dot repositionable electrodes manufactured by $3 \mathrm{M}$ were used in this study. The rectangular electrodes with a contact area of $10.1 \mathrm{~cm}^{2}$ and snap-button connectors, utilize conductive and adhesive hydro gel to ensure correct contact.

\section{ECG and ICG Measurements}

For this study a TEB-measurement one-minute in duration was recorded on three healthy subjects standing in a resting state and following the measurement protocol presented in Fig.2. Using the textrode belt set and conventional $\mathrm{Ag} / \mathrm{AgCl}$ electrodes, single frequency measurements at 50 $\mathrm{kHz}$ were performed with the Respimon impedance cardiograph. The measurements were done using a tetra polar configuration and the lateral spot electrode array as suggested by Woltjer in [12]. The measurement set-up used is represented in Fig. 1A.

\section{Measurement Instrumentation}

Impedance Cardiograph (Respimon). - Contains an impedance plethysmography device that provides 2 EBI

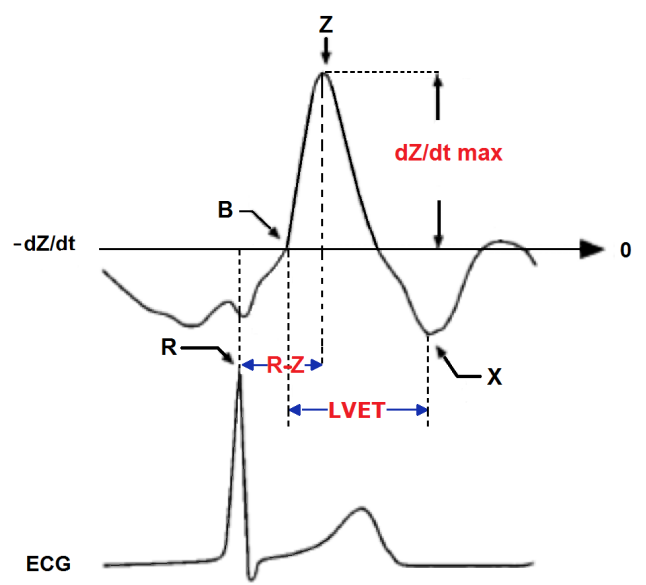

Fig. 3 Analysed ICG and ECG waveforms. The singular point $\mathrm{dZ} /$ dtmax (Z-spot), LVET (time from B to X-spot) and R-Z (time from $\mathrm{R}$ to $\mathrm{Z}$-spot) parameters are indicated. 


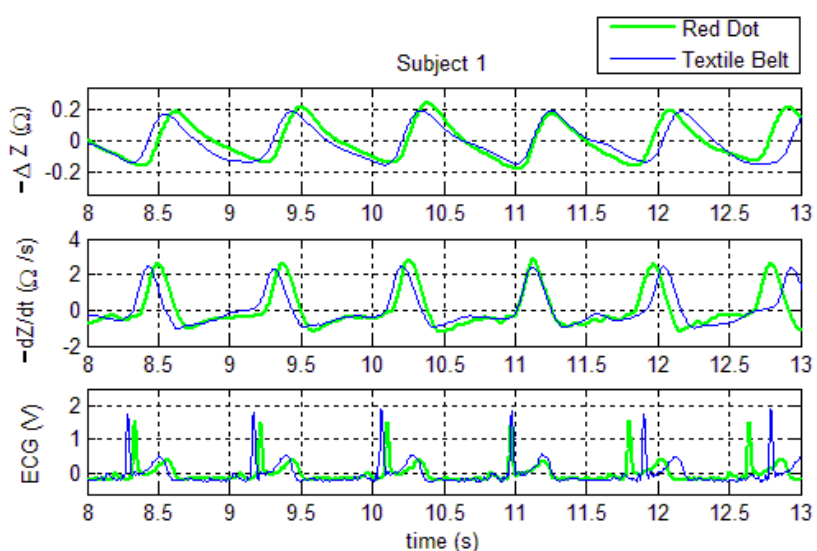

Fig. 4 ICG signals $\Delta \mathrm{Z}$, -dZ/dt and ECG from Subject 1. On green trace the reference $\mathrm{Ag} / \mathrm{AgCl}$ electrode and on blue trace the textile belt.

analog outputs: base impedance $Z_{0}$ and impedance change $\Delta \mathrm{Z}$ from a tetrapolar TEB impedance measurement performed at $50 \mathrm{kHz}$ and a biopotential amplifier that produces and ECG signal.

LabView Data Acquisition Card NI USB-6218. - This card was used to digitize the analogue signals produced by the impedance cardiographer. The card was connected through USB to a PC that recorded and stored the measurements for further processing and analysis with MATLAB.

\section{E. Measurement Analysis and Studied Parameters}

MATLAB Processing. To remove unwanted noise and offset such as $50 \mathrm{~Hz}$ noise and respiration, a digital filtering stage was implemented. Time delay estimation and compensation for the digital filters, signal synchronization, characteristic point detection, parameter estimation and statistical processing were all done in MATLAB.

Analyzed Parameters. - Heart rate estimated from the ICG, ECG, and peak first time derivative $\mathrm{dZ} / \mathrm{dt}_{\max }$ were some of the parameters analyzed in this study. In addition, a relevant hemodynamic parameter stroke volume, as well as the timing parameters left ventricular ejection time (LVET) and $\mathrm{R}-\mathrm{Z}$ were compared.

Left Ventricular Ejection Time (LVET). The time elapsed from the opening to the closure of the aortic valve. In Figure 3 correspond to the time between points B and X.

$R$ to $Z$ time (R-Z). Represents the time span between R-peak in the ECG and the maximal point in the $\mathrm{dZ} / \mathrm{dt}$ curve. In Figure 3 correspond to the time between R and Z. R-Z time is used in the estimation of the Heather Index [13, 14], considered an index of the cardiac contractility.

Stroke Volume Formula. The estimation of stroke volume was done using the equation 1 . In this equation proposed by Bernstein et al $[15,16], \mathrm{V}_{\mathrm{EPT}}$ represents the volume of electrically participating thoracic tissue $(\mathrm{mL}), \mathrm{dZ}(\mathrm{t}) / \mathrm{dt}_{\max }$ the peak of first time derivative $\left(\mathrm{ohm} / \mathrm{s}^{2}\right), \mathrm{Z}_{0}$ the measured
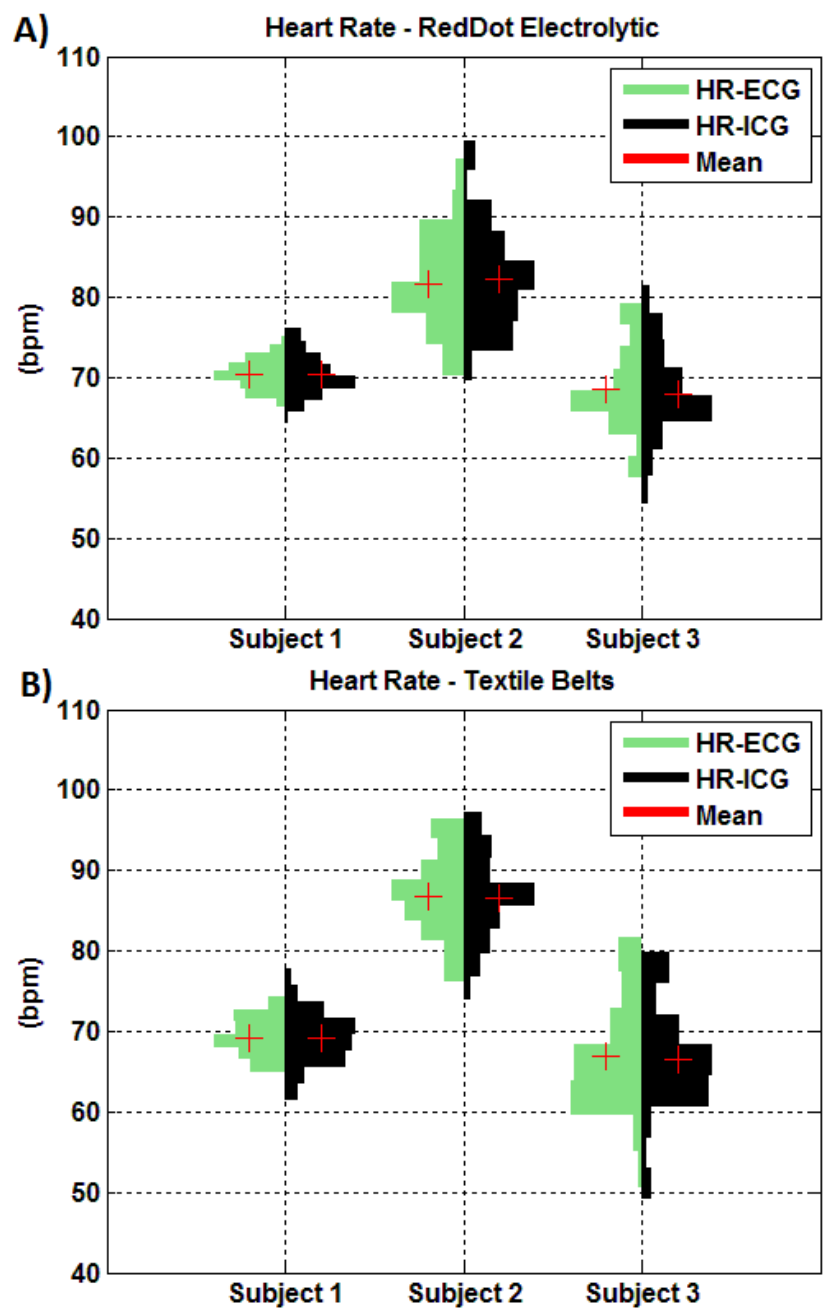

Fig. 5 Distribution plots of the heart rate extracted from the ECG and ICG signals with the corresponding mean values. In (A) HR from electrolytic electrodes and (B) from textile belts.

transthoracic base impedance (ohms) and LVET the left ventricular ejection time (s).

$$
S V=V_{E P T} \sqrt{\frac{d Z(t) / d t_{\max }}{Z_{0}}} L V E T
$$

Performed Comparison. The comparison in this work was based on the signal quality of the ICG and ECG curves and on consistencies in the estimation of relevant parameters. The signals were recorded during one minute and the parameter variation was analyzed by means of mean values and data dispersions.

\section{Measurement Results}

\section{A. ICG Recordings}

In Fig. 4 the fundamental ICG signals impedance change $\Delta \mathrm{Z}, \mathrm{dZ} / \mathrm{dt}$, and ECG corresponding to Subject 1 are presented. From these signals no significant dissimilarities can be observed. The R-peak magnitudes acquired with the textile belts seem to be slightly larger, although this could be attributed to variations of the electrode placement with the two different types of electrodes. 

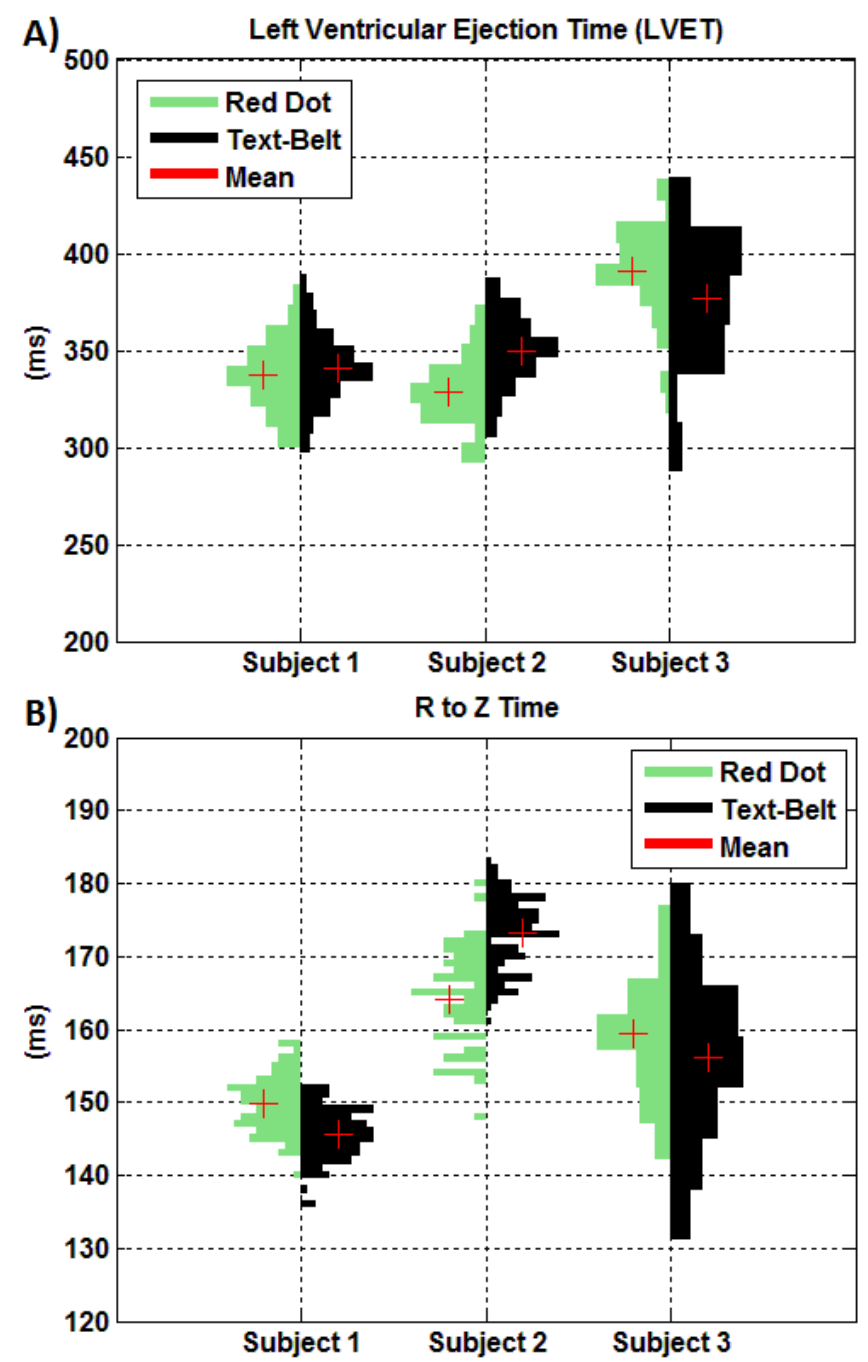

Fig. 6 Estimated time intervals showing the distribution plots and mean values. (A) Left ventricular ejection time and (B) characteristic period $\mathrm{R}$ to $\mathrm{Z}$ time.

\section{B. ICG Parameter Estimation}

The estimated heart rate values obtained from the impedance and the ECG signals are presented in Fig. 5. In this figure, the HRs obtained from the electrolytic electrode were analyzed and compared against the HRs obtained from the textile belts. From Figure 5A and 5B high degree of similarity and minor differences were observed between the HRs derived from the ECG and ICG signals. The two types of electrode, textile and electrolytic, presented similar distributions with no significant differences in mean values and dispersions for any of the subjects.

The results of the time interval LVET and the R to $\mathrm{Z}$ time are presented on Fig. 6. For the LVET in Fig. 6A, a clear overlap of the estimated values and similar distributions were observed for the three subjects with a maximal mean difference of $20.93 \mathrm{~ms}$ for Subject 2. The largest dispersion is observed in the textile-belt for subject 3. In the $\mathrm{R}$ to $\mathrm{Z}$ period shown in Fig. 6B, similar distributions were obtained with both types of electrodes and for the three subjects. Subject 2 presented the most evident mean difference with almost $9 \mathrm{~ms}$ whereas the
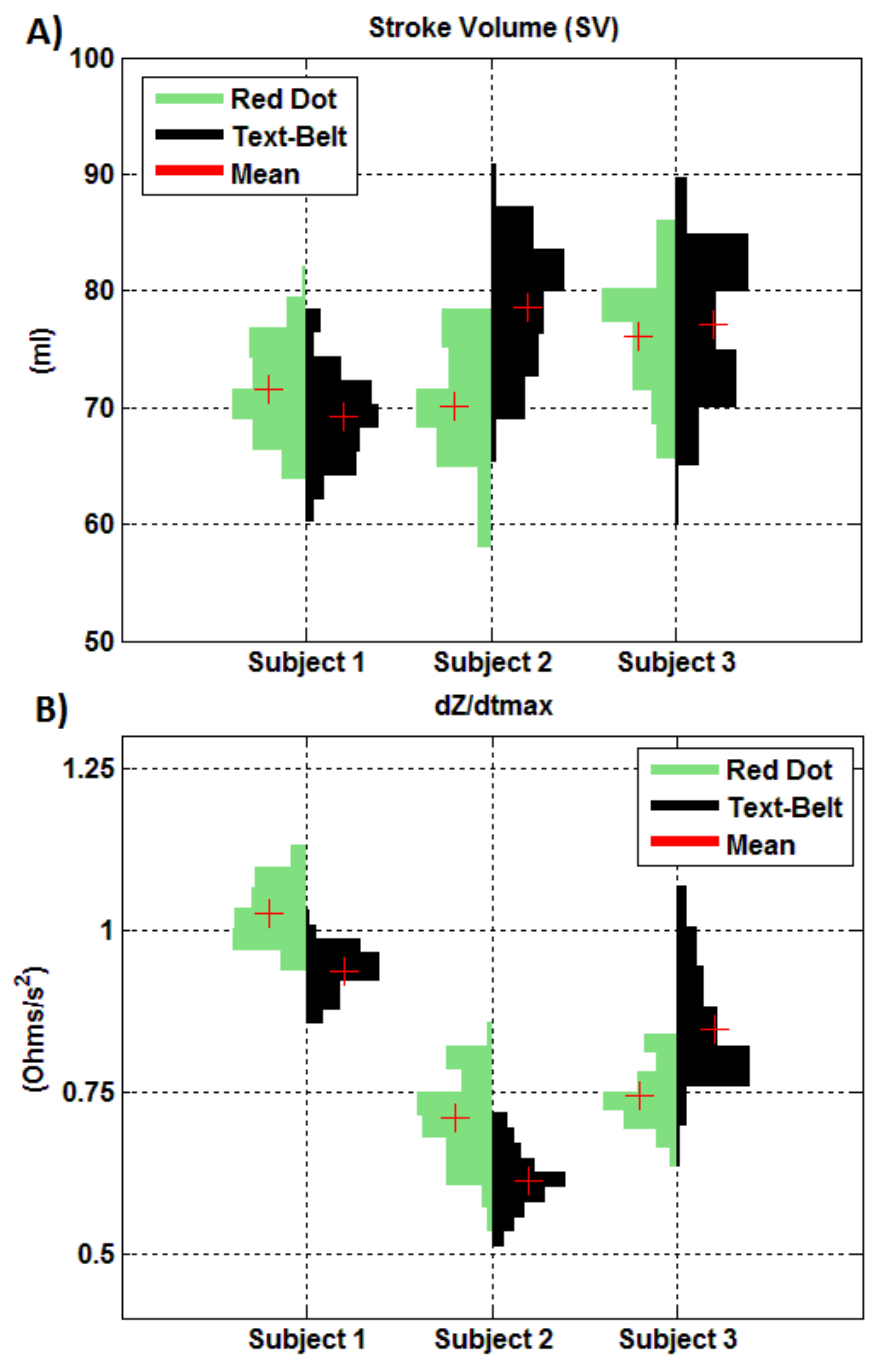

Fig. 7 Estimated parameters showing the distribution plots and mean values (A) Stroke volume estimation based on equation (1) and (B) peak first time derivative $\mathrm{dZ}(\mathrm{t}) / \mathrm{dtmax}$.

largest data variance was observed in the Subject 3 when using the textile belt.

The peak first time derivative $\mathrm{dZ}(\mathrm{t}) / \mathrm{dt}_{\max }$ and the SV estimation are shown in Fig. 7. The SV estimation depicted in Fig. 7A presented overlapping zones in the distribution plots for the three subjects. Subject 3 showed a very close mean value but also the largest dispersion when using textile-belt. On the other hand Subject 2 exhibited the largest mean difference of $8.60 \mathrm{ml}$. The peak first time derivative $\mathrm{dZ}(\mathrm{t}) / \mathrm{dt}_{\max }$ also presents evident overlapped zones of the distribution plots for all the subjects. In Fig. 7B) it can be observed how the largest mean difference is found in Subject 3 with $0.11 \mathrm{ohms} / \mathrm{s}^{2}$ and also the largest data dispersion is observed in the same subject with the textrode belt.

\section{Analysis \& Discussion}

This study did not aim to quantify the validity or precision of the parameter for any specific medical condition but to evaluate the performance of textrodes for ICG based on the estimation of relevant parameters and ICG curves. 


\section{A. ICG Curves and HR Estimation}

The ICG waveforms shown in Fig. 4, in general, exhibit good resemblance. Differences in the amplitude of the Rpeaks depend more on the exact position of the electrodes than the electrode type. The time shifting observed are caused by the natural heart rate variability rather than by the electrode difference. The small differences observed in the HR analysis extracted from both sources (ECG and ICG) are most probably attributed to the natural HR variability of different measurement sessions and not necessarily to the type of electrode. Measurements obtained with both types of electrodes presented reasonable values of HR according to [17]. There were no missing beats and no need for special signal processing techniques for the signals acquired with belts. Thus the same algorithms for processing and detection were used for both types of electrodes.

\section{B. Stroke Volume Equation}

Stroke volume is probably one of the most medical relevant hemodynamic indices obtained using thoracic electrical bioimpedance $[18,19]$. Despite the clinically attractive attributes of SV estimation by means of TEB, the method has shown low consistency between the impedance wave forms and hemodynamic variables [20]. This inefficiency in the correlation between curves and estimated variables suggest a lack of robustness in the equation models to estimate SV and a low sensitivity and specificity of the impedance signal acquisition in some medical conditions. In addition to this, a necessary consensus on whether the physiological origin of $\mathrm{dZ} / \mathrm{dt}$ comes predominantly from volume changes as traditionally believed or from blood resistivity changes in the vessels as more recent evidence presented by Bernstein et al. $[16,21]$ indicates must still be arrived at.

\section{Stroke Volume \& $d Z(t) / d t_{\max }$ Estimation}

The peak value $\mathrm{dZ}(\mathrm{t}) / \mathrm{dt}_{\max }$ is an important parameter in the estimation of stroke volume and other parameters. The peak first derivative measurements done with the belts presented a reasonable level of consistency. As can be seen from Table I, measurements performed with the textrode belts,

TABLE I. ICG PARAMETERS

\begin{tabular}{ccccccccccc}
\hline \hline & & \multicolumn{2}{c}{$\begin{array}{c}\text { dZ/dt max } \\
\left(\Omega / \mathrm{s}^{2}\right)\end{array}$} & \multicolumn{2}{c}{$\mathrm{SV}$} & \multicolumn{2}{c}{ LVET } & \multicolumn{2}{c}{ R-Z Time } \\
$(\mathrm{ml})$ & \multicolumn{2}{c}{$(\mathrm{ms})$} & \multicolumn{2}{c}{$(\mathrm{ms})$} \\
& & avg & std & avg & std & avg & std & avg & std \\
\hline & & & & & & & & & \\
S1 & RedDot & 1.03 & 0.05 & 71.52 & 3.94 & 336.99 & 17.34 & 149.78 & 3.87 \\
& Tex-Belt & 0.94 & 0.03 & 69.15 & 3.63 & 341.07 & 18.41 & 145.60 & 3.54 \\
& & & & & & & & & \\
S2 & RedDot & 0.71 & 0.07 & 70.04 & 4.88 & 328.58 & 16.92 & 164.20 & 6.54 \\
& Tex-Belt & 0.61 & 0.05 & 78.64 & 5.28 & 349.51 & 17.77 & 173.14 & 5.04 \\
& & & & & & & & & \\
S3 & RedDot & 0.74 & 0.05 & 76.03 & 4.62 & 391.11 & 22.36 & 159.33 & 7.73 \\
& Tex-Belt & 0.85 & 0.09 & 77.07 & 6.20 & 376.43 & 32.93 & 156.25 & 10.97 \\
\hline \hline
\end{tabular}

N.B. For detailed information regarding SV estimation see references 14 and 15. showed a mean variation from $85.9 \%$ to $114.8 \%$ of the values obtained from the $\mathrm{Ag} / \mathrm{AgCl}$ reference electrode. Similar behavior was observed for the SV estimation where mean values of the textrode belts shown differences from $96.7 \%$ to $112.2 \%$ of the values acquired with the reference. No evident trends related with the type of electrode were found in the estimation of these two parameters. However, since measurements with the two types of electrodes were not taken simultaneously but rather sequentially in a different measurement session, variations in the SV estimation may not be entirely attributed to the type of electrode used but instead to the natural biological differences between two measurement sessions. Nonetheless SV measurements obtained with both type of electrodes can be considered as physiologically normal according to [22].

\section{Left Ventricular Ejection \& $R$ to $Z$ time}

The timing parameters estimated with the textrode belts presented relative close resemblance. The LVET values obtained by the textrode belts and presented in Table I appear reasonable, ranging from 341 to $376 \mathrm{~ms}$. Similar measurements were obtained and reported by Cokkinos et al [23]. Indeed the values obtained with the belt represent $96.2 \%$ to $106.3 \%$ of the value measured with the corresponding $\mathrm{Ag} / \mathrm{AgCl}$ electrode.

A similar performance was observed in the estimation of $\mathrm{R}$ to $\mathrm{Z}$ time where measurements fluctuated from $97.2 \%$ to $105.4 \%$ of the corresponding value. This difference seems to be very small and although such timing parameters can be used to estimate hemodynamic information, the influence of observed differences on the estimation of such hemodynamic indices, was not analyzed in this study.

\section{Conclusions}

As a first step to evaluate the performance of textile electrodes for TEB measurements to obtain cardioimpedance recordings, the analysis of ICG recordings, time period LVET, characteristic period $\mathrm{R}$ to $\mathrm{Z}$ and left ventricular stroke volume performed in this work, did not showed any specific trend regarding the type of electrode utilized but it establishes a good starting point towards a more robust assessment of the use of textile sensors.

Determining if the parameters estimated with the textrode belts produce accurate and reliable enough results is highly dependent on the clinical application or individual case, for instance an error rate of $10 \%$ in the HR may be well acceptable for some applications while in others one, $0.5 \%$ may be not acceptable for an accurate diagnostic or may not be useful to differentiate between healthy and disease.

In any case for both the waveforms and the calculated parameters, the resemblance is close and the measurements 
obtained produced reasonable and physiologically normal parameters for healthy subjects at resting state.

In spite of the encouraging results and resemblance of the estimation of timing and systolic parameters done in this study, further analysis is required in order to determine the implications of using textile electrodes for the determination of hemodynamics indices.

\section{References}

1. Li G, Geng Y, Tao D, Zhou P. Performance of electromyography recorded using textile electrodes in classifying arm movements. Conf Proc IEEE Eng Med Biol Soc. 2011:4243-6.

2. Pola T, Vanhala J, editors. Textile Electrodes in ECG Measurement. Intelligent Sensors, Sensor Networks and Information ISSNIP 2007 3-6 Dec.

3. Márquez JC, Seoane F, Välimäki E, Lindecrantz K. Comparison of dry-textile electrodes for electrical bioimpedance spectroscopy measurements. Journal of Physics: Conference Series. 2010;224(1):012140. http://dx.doi.org/10.1088/1742-6596/224/1/012140

4. Marquez JC, Ferreira J, Seoane F, Buendia R, Lindecrantz K. Textile electrode straps for wrist-to-ankle bioimpedance measurements for Body Composition Analysis. Initial validation \& experimental results. Conf Proc IEEE Eng Med Biol Soc; Buenos Aires, Argentina2010. p. 6385-8.

5. Centers for Medicare \& Medicaid Services. National Coverage Determination (NCD) for Cardiac Output Monitoring by Thoracic Electrical Bioimpedance (TEB). In: (DHHS) DoHHS, editor.: Department of Health \& Human Services (DHHS); 2006.

6. Seoane F, Rempfer M, Marquez JC, Lindecrantz K. Textileenabled Instrumentation for Impedance Cardiographic Signals. Medicinteknikdagarna; October 11-12,; Linköping, Sweden 2011

7. Geddes LA, Baker LE. The specific resistance of biological material - a compendium of data for the biomedical engineer and physiologist. Med Biol Eng. 1967;5(3):271-93. http://dx.doi.org/10.1007/BF02474537

8. Geddes LA, Sadler C. The specific resistance of blood at body temperature. Med Biol Eng. 1973;11(3):336-9. http://dx.doi.org/10.1007/BF02475543

9. Lababidi Z, Ehmke DA, Durnin RE, Leaverton PE, Lauer RM. The first derivative thoracic impedance cardiogram. Circulation. 1970;41(4):651-8. http://dx.doi.org/10.1161/01.CIR.41.4.651

10. Kamath SA, Drazner MH, Tasissa G, Rogers JG, Stevenson LW, Yancy CW. Correlation of impedance cardiography with invasive hemodynamic measurements in patients with advanced heart failure: the BioImpedance CardioGraphy (BIG) substudy of the Evaluation Study of Congestive Heart Failure and Pulmonary Artery Catheterization Effectiveness (ESCAPE) Trial. Am Heart J. 2009;158(2):217-23. http://dx.doi.org/10.1016/j.ahj.2009.06.002

11. Trakic A, Akhand M, Wang H, Mason D, Liu F, Wilson S, et al. Computational modelling of blood-flow-induced changes in blood electrical conductivity and its contribution to the impedance cardiogram. Physiol. Meas. 2010;31(1):13-33. http://dx.doi.org/10.1088/0967-3334/31/1/002

12. Woltjer HH, Bogaard HJ, Scheffer GJ, van der Spoel HI, Huybregts MA, de Vries PM. Standardization of non-invasive impedance cardiography for assessment of stroke volume: comparison with thermodilution. Br J Anaesth. 1996;77(6):748-52. http://dx.doi.org/10.1093/bja/77.6.748

13. Heather LW. Development and evaluation of an Impedance Cardiographic system to measure Cardiac Output and other carsdiac parameters. University of Minnesota Collage of Medical Science, Minneapolis, Minnesota: National aeronautics and Space administration NASA, 1969 Contract No.: 19700000711.

14. Patterson RP. Fundamentals of impedance cardiography. Engineering in Medicine and Biology Magazine, IEEE. 1989;8(1):35-8. http://dx.doi.org/10.1109/51.32403

15. Bernstein DP, Lemmens HJ. Stroke volume equation for impedance cardiography. Med Biol Eng Comput. 2005;43(4):443-50. http://dx.doi.org/10.1007/BF02344724

16. Bernstein DP. Impedance cardiography: Pulsatile blood flow and the biophysical and electrodynamic basis for the stroke volume equations. Journal of Electrical Bioimpedance. 2010;1:2-17.

17. Palatini P. Need for a revision of the normal limits of resting heart rate. Hypertension. 1999;33(2):622-5.

18. Moshkovitz Y, Kaluski E, Milo O, Vered Z, Cotter G. Recent developments in cardiac output determination by bioimpedance: comparison with invasive cardiac output and potential cardiovascular applications. Curr Opin Cardiol. 2004;19(3):229-37. http://dx.doi.org/10.1097/00001573-200405000-00008

19. Summers RL, Shoemaker WC, Peacock WF, Ander DS, Coleman TG. Bench to bedside: electrophysiologic and clinical principles of noninvasive hemodynamic monitoring using impedance cardiography. Acad Emerg Med. 2003;10(6):669-80. http://dx.doi.org/10.1111/j.1553-2712.2003.tb00054.x

20. Kauppinen PK, Hyttinen JA, Malmivuo JA. Sensitivity distributions of impedance cardiography using band and spot electrodes analyzed by a three-dimensional computer model. Ann Biomed Eng. 1998;26(4):694-702. http://dx.doi.org/10.1114/1.44

21. Bernstein DP, Henry IC, Banet MJ, Dittrich T. Stroke volume obtained by electrical interrogation of the brachial artery: transbrachial electrical bioimpedance velocimetry. Physiol. Meas. 2012;33(4):629-49. http://dx.doi.org/10.1088/0967-3334/33/4/629

22. Lifesciences E. Normal Hemodynamic Parameters and Laboratory Values. Edwards; 2011; Available from: http://ht.edwards.com/scin/edwards/sitecollectionimages/ edwards/products/presep/ar05688_parameters.pdf.

23. Cokkinos DV, Heimonas ET, Demopoulos JN, Harralambakis A, Tsartsalis G, Gardikas CD. Influence of heart rate increase on uncorrected pre-ejection period/left ventricular ejection time (PEP/LVET) ratio in normal individuals. Br Heart J. 1976;38(7):683-8. http://dx.doi.org/10.1136/hrt.38.7.683 